\title{
Broadening Risk Profile in Familial Colorectal Cancer Type X; increased risk for five cancer types in the national Danish cohort
}

Christina Therkildsen ( $\sim$ christina.therkildsen@regionh.dk)

Copenhagen University Hospital, Hvidovre https://orcid.org/0000-0002-2833-6986

Maria Rasmussen

Hvidovre Hospital

Lars Smith-Hansen

Hvidovre Hospital

Thomas Kallemose

Hvidovre Hospital

Lars Joachim Lindberg

Hvidovre Hospital

Mef Nilbert

Kraeftens Bekaempelse

Research article

Keywords: Tumour spectrum, Hereditary cancer, Cancer syndrome, Mismatch repair proficient, Amsterdam I criteria

Posted Date: January 8th, 2020

DOI: https://doi.org/10.21203/rs.2.20266/v1

License: (c) (1) This work is licensed under a Creative Commons Attribution 4.0 International License. Read Full License

Version of Record: A version of this preprint was published at BMC Cancer on April 22nd, 2020. See the published version at https://doi.org/10.1186/s12885-020-06859-5. 


\section{Abstract}

\section{Background}

Familial colorectal cancer type X (FCCTX) is a phenotypically defined subset of hereditary colorectal cancer with unknown and potentially heterogeneous genetic aetiology. Though FCCTX has been characterized as a colorectal cancer-specific syndrome, we aimed to estimate the risk for extra-colorectal cancer in the Danish FCCTX cohort.

\section{Methods}

Through the national hereditary non-polyposis colorectal cancer (HNPCC) register, 213 families fulfilling the Amsterdam I criteria and showing retained mismatch repair (MMR) function were identified. In here, sex and age-specific incidence rate ratios (IRR) were calculated for 30 extra-colorectal cancer types in comparison with the general Danish population.

\section{Results}

In total, 494 extra-colorectal cancers developed with significantly increased risks for the urinary tract, breasts, stomach, pancreas, and eye. The age spans, during which increased risks were observed, were 30-49 years for gastric cancer, 30-69 years for female breast cancer, 50-69 years for ocular melanoma and above age 70 for pancreatic cancer and urothelial cancer.

Conclusions

These risk estimates suggest an increased risk of several extra-colorectal cancer types, which calls for awareness during genetic counselling and follow-up. The cancers that occur at increased risk may indicate unidentified disease-predisposing genetic variants in this phenotypically defined subset of hereditary colorectal cancer.

\section{Introduction}

Heredity is estimated to explain $~ 20 \%$ of the colorectal cancer diagnoses and covers a complex genetic landscape (Valle, 2017). Though several rare high-risk alleles have been identified, a large fraction of families with seemingly inherited colorectal cancer diagnoses remains genetically undefined. In here families, who meet the Amsterdam I criteria for Lynch syndrome, but with no signs of mismatch repair (MMR) deficiency, i.e. a mismatch-repair stable phenotype and/or retained MMR protein expression, are referred to as familial colorectal cancer type X (FCCTX) (Lindor et al., 2005, Vasen et al., 2013). This subset constitutes $40 \%$ of the families that fulfil the Amsterdam I criteria and belong to the hereditary non-polyposis subgroup of hereditary cancer (Valle, 2014). The genetic aetiology of FCCTX is most likely heterogenous and may include pathogenic variants in e.g. MUTYH, BRCA2, POLE, POLD1, SEMA4A, BMPR1A, BMP4, RSP20, NTHL1, or RNF43, modifying genes or polygenic components (Francisco et al., 
2011; Ku et al., 2012; Nejadtaghi et al., 2017; Villacis et al., 2016; Warden et al., 2013; Zetner and Bisgaard, 2017).

Besides the MMR proficient molecular phenotype, FCCTX-associated colorectal cancers have been distinguished from the genetically defined cancer syndrome, Lynch syndrome, by a predilection for tumour development in the distal colon and the rectum, a high adenoma/carcinoma rate and a lower risk of synchronous and metachronous colorectal cancer (Dove-Edwin et al., 2006; Klarskov et al., 2012; Mueller-Koch et al., 2005; Shiovitz et al., 2014). The risk of colorectal cancer is lower than in Lynch syndrome with a relative risk (RR) of 0.5 , but higher than the general population with a standardized incidence ratio (SIR) of 2.3 (Benatti et al., 2001, Lindor et al., 2005). Current literature suggests that FCCTX is a colorectal cancer-only syndrome, which provide the basis for current recommendations of surveillance with regular colonoscopy starting 5-10 years prior to the youngest case in the family in families classified as FCCTX (Benatti et al., 2001; Lindor et al., 2005; Mueller-Koch et al., 2005; Valle et al., 2007).

We challenged this notion through risk assessment of 30 different extra-colorectal cancer types in the Danish FCCTX cohort compared to the Danish general population and to the national Lynch syndrome cohort and found increased risk of five extra-colorectal cancer types, i.e. urothelial cancer, female breast cancer, gastric cancer, pancreatic cancer, and ocular melanoma.

\section{Materials And Methods}

The Danish hereditary non-polyposis colorectal cancer (HNPCC) register contains 6000 families with suspected or verified hereditary colorectal cancer reported to the register by genetic counsellors, surgeons, pathologists, and genetic diagnostic laboratories. Families have been included based on a suspicious family history of colorectal cancer, fulfilment of the Amsterdam I or II criteria (Vasen et al., 2013), or identification of disease-predisposing variants in genes linked to hereditary colorectal cancer. Based on family history the register subclassifies families according to genotypic and phenotypic subsets (Lindberg et al., 2017). The Danish HNPCC register identifies all family members in the Danish Civil Registration System, regardless of cancer history, based on data collected from clinical files and health care registers.

\section{Patient selection}

Families classified as FCCTX $(n=213)$ were eligible for the study. FCCTX was defined as fulfilment of the Amsterdam I criteria with no signs of MMR deficiency. The Amsterdam I criteria are defined as at least three relatives with histologically verified colorectal cancer in two generations with one individual being a first-degree relative of the other two and at least one individual diagnosed below the age of 50 (Vasen et al., 2013). A maximum of one tumour with MLH1/PMS2 loss was accepted if this was in conjunction with a BRAF mutation and/or MLH1 promotor hypermethylation $(\mathrm{N}=4)$ or normal MMR expression was found in $\geq 1$ tumour from a family member $(\mathrm{N}=16)$. Loss of MSH2/MSH6 protein expression was not allowed, while loss of MSH6 only was observed in two cases and allowed motivated by normal genetic 
test result in the same individual $(\mathrm{N}=1)$ or normal MMR protein expression in another tumour in the same family $(N=1)$. Variants of unknown significance were included only when normal MMR protein expression was verified in a tumour from the same patient $(\mathrm{N}=2)$. Of the 252 Amsterdam I positive families reviewed, 213 fulfilled the criteria for MMR proficiency. Individuals affected with colorectal cancer and their first- and second-degree relatives were eligible for the study.

\section{Data processing}

Data on primary extra-colorectal cancer diagnoses were obtained from the population-based Danish Cancer Registry. This registry has close to complete coverage based on mandatory double reporting from pathologists and clinicians (Storm, 1988; Storm et al., 1997). Benign tumours, carcinoma in situ/dysplasia, and basal cell carcinomas of the skin were excluded. Patients with more than one primary cancer in different organs were allowed to contribute to the tissue-specific risk estimates, while synchronous/metachronous cancer in the same organ or in the same side of paired organs were not allowed. Data on vital status were obtained from the Danish Civil Registration System.

To determine the risk relative to the general population, we used a population-based cohort obtained from the Nordcan database (Engel G. et al., 2016). This cohort contains data on age-specific cancer events and person years at-risk in the Danish background population during the time period from January 1st, 1978 to December 31st, 2013 with stratification for year of diagnosis, sex, age, and disease. The Nordcan database classifies malignancies into 36 groups. The FCCTX-associated cancers could be matched to 30 of these after exclusion of cancer in the colon, rectum and anal canal, unspecified cancers, specified cancer (grouped by Nordcan), and 2 rare specified malignancies without cases in the FCCTX cohort. All cancers and person years at-risk identified in the Danish FCCTX cohort and the previously published Danish Lynch syndrome cohort were removed from the Nordcan data set (Therkildsen et al., 2017). To correct for potential ascertainment bias, we performed a subgroup analysis in a colorectal cancersurveilled cohort, reflecting prospective data, with inclusion of cancers diagnosed following the first colonoscopic surveillance session in the family and exclusion of diagnoses and person years at-risk prior to this date. The study was granted acceptance from the Danish Data Protection Agency. According to Danish regulations, registry studies should not be subject to ethical review.

Statistical analyses

Person years at-risk and cancer events in the FCCTX cohort and in the population-based Nordcan cohort were stratified and aggregated into 4 age groups (0-29 years, 30-49 years, 50-69 years and 70 years or above) using the \%STRATIFY SAS macro, which removes individuals from the at-risk group if cancer is diagnosed within the study period, and SAS software, version 9.4 (SAS Institute Inc., Cary, NC, USA) (Rostgaard, 2008). Person years at-risk were determined as the period from date of birth or start of study period (January 1st, 1978), whichever came last, to date of diagnosis of any type of cancer, date of death or end of study period (December 31st, 2013), whichever came first. 
Stratified and aggregated data were transferred into R 3.2.3 (R Core Team, 2019). Incidence rates (IRs) were calculated as the number of events divided by person years at-risk in each age group. Incidence rate ratios (IRRs) were calculated as the ratio between the IRs in the FCCTX cohort and the population-based cohort. Since FCCTX is part of the HNPCC subgroup of hereditary colorectal cancer and are classified according to the same clinical criteria, i.e. the Amsterdam criteria, we also estimated IRs relative to the previous IRs published in the Danish Lynch syndrome cohort (Therkildsen et al., 2017). Confidence intervals $(95 \% \mathrm{Cl})$ and $p$ values were calculated using the exact conditional Poisson test. All $p$ values were two-sided and significance levels were adjusted for multiple testing using Bonferroni correction for estimation in the 4 age groups (i.e. significance was reached when $p<0.0125$ ).

\section{Results}

The FCCTX cohort comprised 213 families, including 646 individuals with a colorectal cancer, 1,982 firstdegree relatives and 1,044 second-degree relatives. These individuals contributed with 110,767 personyears at-risk and during this time 966 individuals developed 1,079 cancers, including 494 extra-colorectal cancers and 585 colorectal cancers (Supplementary table 1). The most prominent cancer types observed were breast cancer $(N=104)$, prostate cancer $(N=51)$, urothelial cancer $(N=45)$, and lung cancer $(N=40)$. Prospective analyses were based on 223 individuals in surveillance from 109 families, who contributed with 903,448 person years at-risk. In this subgroup that considered only cancers that developed after the initiation of surveillance in the family, 160 cancers, including 26 breast cancers, 21 urothelial cancers and 19 prostate cancers, were diagnosed (Supplementary table 1).

Compared to a population-based cohort, the FCCTX cohort revealed significantly increased risks for five cancer types, i.e. breast cancer, urothelial cancer, pancreatic cancer, gastric cancer, and ocular melanoma with variable peak incidence ages identified in the different tumour types (Fig. 1, Supplementary table 2). Significantly increased IRRs were observed for breast cancer in the age groups from age 30 until 69 years (IRR for age 30-49: 1.71, 95\% Cl 1.02-2.68, p = 0.0070 and IRR for age 50-69: 1.54, 95\% Cl 1.06-2.14, $p$ $=0.0030)$, while gastric cancer showed an IRR of 5.87 (95\% Cl 1.57-15.02, p = 0.0007) for the age group 30-49 years. Urothelial cancer and pancreatic cancer developed at significantly increased IRRs of 2.10 (95\% Cl 1.26-3.30, p = 0.0003) and 2.21 (95\% Cl 1.12-4.38, p = 0.0023), respectively, in the oldest age cohort, above age 70. In total, five eye tumours developed in age group 50-69 years, all of which were classified as ocular melanomas, giving an IRR of $7.73(95 \% \mathrm{Cl} 1.76-21.45, \mathrm{p}=0.0006)($ Fig. 1, Table 1). In the prospective analysis, significantly increased risks applied to urothelial cancer and ocular melanoma with an IRR of $2.94(95 \% \mathrm{Cl} 1.34-5.54, \mathrm{p}=0.0004)$ above age 70 for urothelial cancers and an IRR of $13.81(95 \%$ Cl 1.68-49.32, $p=0.0015)$ for ocular melanomas (Table 2)

The increased risks of extra-colorectal cancers applied to all FCCTX individuals with similar risk levels in first-degree and second-degree relatives compared to the relatives affected with colorectal cancer (Supplementary Fig. 1). 
Compared to Lynch syndrome, FCCTX families showed significantly lower risks in at least one age group for eight different tumour types, i.e. endometrial cancer, ovarian cancer, urothelial cancer, kidney cancer, gastric cancer, cancer of the small bowel, non-melanoma skin tumours and brain tumours (Table 3). For urothelial cancer and skin cancer this observation was consistent from age 30+. The risk of urothelial cancer in FCCTX showed IRRs of 0.06-0.31 ( $p$-values < 0.0009) compared to Lynch syndrome and the risk of skin cancer showed IRRs of $0.11-0.24(p<0.005)$ (Table 3$)$. For the other tumour types this difference particularly applied to the peak ages of these cancer types in Lynch syndrome, i.e. age 3069 years (Table 3 ). No difference in risks of breast cancer, eye tumours and pancreatic cancer were found between the FCCTX and Lynch syndrome cohorts for all age groups (Supplementary table 4).

\section{Discussion}

Increased awareness of hereditary colorectal cancer and improved access to genetic diagnostics implies that a growing number of families with a phenotype suggesting hereditary cancer with an undefined genotype have been identified. FCCTX represents one of these subsets where refined risk estimates are relevant to develop evidence-based surveillance recommendations. Lindor et al., described a standardized incidence ratio for colorectal cancer of 2.3 in a cohort of 71 FCCTX families and did not identify any significantly increased risk of extra-colorectal cancer (Lindor et al., 2005). Based on this observation, FCCTX is considered a colorectal cancer-only syndrome with surveillance generally recommended to be confined to colonoscopy with 5-year intervals starting 5-10 years prior to the first case in the family. Surveillance programs for colorectal cancer have been optimized with documentation of more efficient detection of precursor lesions and early-stage tumours (RR 0.2-0.3) (Hatfield et al., 2018). Reduced risk of mortality from colorectal cancer and increased life expectancy implies that individuals with FCCTX may be at risk of extra-colorectal tumour types during this increased life-time. Our data, based on all 213 FCCTX families in the national Danish HNPCC-register, challenges the present view on FCCTX as a colorectal cancer-only syndrome and demonstrate significantly increased incidence rates for five extracolorectal cancer types with urothelial cancer remaining significant in the colorectal cancer-surveilled cohort (Fig. 1, Table 1).

We demonstrated an increased risk of urothelial cancer from age 70 in FCCTX with IRRs of 2.1 in the entire FCCTX cohort and 2.9 in the surveilled subset compared to the risk in an age- and sex matched Danish population (Tables 1 and 2). The risk of urothelial cancer in FCCTX was significantly lower than in Lynch syndrome with IRRs of 0.1-0.3 (Table 3). Except for Lynch syndrome, urothelial cancer has not been linked to hereditary colorectal cancer (Gu and Wu, 2011). One possibility would be undiagnosed Lynch syndrome cases e.g. MMR gene variants that allow for retained MMR function and normal MMR protein expression. Alternatively, a subset of FCCTX could harbour mutations in genes linked to urothelial cancer development, e.g. FGFR3, TP53 or HRAS (Zhang et al.,2015). To this point, 42/45 urothelial cancer in the FCCTX cohort developed in the urinary bladder, which stands in contrast to a predilection for tumours in the upper urinary tract in Lynch syndrome. 
The increased risk of gastric cancer with an IRR of 5.9 in the age group 30-49 years could potentially signify a genetic subset that confers heredity for this cancer type. About $10-20 \%$ of gastric cancer is caused by heredity with confirmed causes in $1-3 \%$, predominantly linked to the hereditary diffuse gastric cancer caused by $\mathrm{CDH} 1$ (van der Post et al., 2019). Criteria for genetic testing do in western countries consider development of diffuse gastric cancer before age 40-50 years in conjunction with lobular breast cancer or development of intestinal gastric cancer in at least two first- or second-degree relatives and diagnosis before the age of 50 years (Chun and Ford, 2012; van der Post et al., 2015). Only two of the 18 FCCTX families in our cohort in which gastric cancer developed fulfilled the criteria currently applied for genetic diagnostics due to early-onset cases in the families (data not shown).

The increased risks of breast cancer with IRRs 1.5-1.7 in the age group 30-69 years and pancreatic cancer with an IRR of 2.2 after age 70 support the suggestion of disease-predisposing variants in BRCA2, causing the observed malignancies in a small subset of FCCTX families (Garre et al., 2015). We also identified an IRR of 10.2 for early-onset ovarian cancer. Various guidelines exist for referring individuals to genetic diagnostics in hereditary breast and ovarian cancer (Zeichner et al., 2016). If the criteria that consider breast cancer before age 45 , more than one primary breast cancer, minimum two breast cancers in the family and development also of epithelial ovarian cancer, prostate cancer and/or pancreatic cancer, 35 of the 85 families in the Danish FCCTX cohort fulfilled at least one of these criteria (data not shown). Though the families included in this study has not been systematically screened for hereditary breast and ovarian cancer, our data support a role for hereditary breast and pancreatic cancer in FCCTX, and application of broader diagnostic genetic panels that also cover the BRCA2 gene may, based on our data, have a potential to identify disease-predisposing mutations in some FCCTX families.

The five eye tumours identified in the Danish FCCTX cohort were all malignant melanomas. Uveal melanoma is predominantly sporadic but between $2-5 \%$ have been estimated to be caused by familial or hereditary predisposition. Autosomal dominant inheritance of pathogenic BAP1 gene variants have been observed in $47 \%$ of uveal melanomas, while pathogenic EIF1AX variants have been found in $14-20 \%$ of the cases (Harbour et al., 2010; Helgadottir and Höiom, 2016). BAP1-associated uveal melanomas are diagnosed in the age of 30-59 years and are associated with cutaneous melanomas and renal cell carcinomas, while EIF1AX gene variants are associated with thyroid and ovarian cancer (Harbour et al., 2010; Helgadottir and Höiom, 2016). In our cohort, uveal melanomas presented in the age span from 5469 years and 2 of 5 cases occurred in patients with previous cutaneous melanomas. These data encourage awareness of family history during the genetic counselling and diagnostic testing.

Comparison between the risk of extra-colorectal cancer in the FCCTX cohort with the national Danish Lynch syndrome cohort, revealed differences as well as similarities. Urothelial cancer and skin cancer showed significantly lower risk levels in FCCTX compared to Lynch syndrome with IRRs of 0.06-0.31 and $0.11-0.24$, respectively (Table 3 ). Increased risks and reminiscent risk profiles applied to breast cancer, gastric cancer and pancreatic cancer. These similarities are also supported by other studies on the risk of upper gastrointestinal cancer in Lynch syndrome (Engel et al., 2012; Kastrinos et al., 2009; Win et al., 2012). Surveillance for cancer of the upper gastrointestinal tract is not recommended in FCCTX, but the 
increased risk observed may suggest awareness with consideration of Helicobacter Pyroli screening and eradication in FCCTX similarly to the recommendations in Lynch syndrome (Ishaq and Nunn, 2015; Vasen et al., 2013; Win et al., 2012).

Studies have shown that compared to Lynch syndrome, FCCTX confers a lower risk for colorectal cancer $(11-20 \%$ vs $58-75 \%)$, a higher age at onset ( 60 vs 45 years), a different predominant tumour locations (distal vs proximal) and a worse prognosis (Choi et al., 2018; Lindor et al., 2005; Nejadtaghi et al., 2017; Zetner and Bisgaard, 2017). In FCCTX, colonoscopic screening is generally recommended with 5-year intervals starting 5-10 years before the earliest colorectal cancer diagnosis in the family, though surveillance patterns are likely more variable in FCCTX than in Lynch syndrome. The recent demonstration of excess cancer-related deaths in FCCTX compared to Lynch syndrome and short intervals to second primary colorectal cancer suggests that clinical management in FCCTX needs to be optimized (Choi et al., 2018).

Our observation of increased risks with distinct and variable incidence patterns in relation to age for five extra-colorectal cancer types in FCCTX needs validation but challenges the present view of FCCTX as a colorectal cancer-only syndrome. The consistently increased risk of urothelial cancer motivates further investigation to obtain more detailed insights into risk profiles and tumour types with the aim to identify possible disease-predisposing genes. The demonstration of increased risks for breast cancer and pancreatic cancer could suggest that genetic variants in BRCA2 may explain some FCCTX families. The FCCTX cohort may be a suitable target for application of broader panels during genetic diagnostics. Further characterization and subdivision of FCCTX are needed to define discriminatory features, provide more robust risk estimates and recommend relevant and cost-effective surveillance to individuals at increased risk.

\section{Declarations}

Author contribution: MN and CT were responsible for the study design and wrote the first draft. LJL contributed to the concept and collected data from the Danish Cancer Society and verified all the tumour diagnoses in the cohort. LSH processed the data in SAS including the \%STRATIFY macro analyses. CT and TK performed the statistical analyses in R. MR commented on the manuscript and assisted with the statistical analyses. All authors have approved the final version and agreed with submission to the British Journal of Cancer.

Funding: This work was supported by grants from the Danish Cancer Research Fund and the Swedish Cancer Research Fund.

Ethics approval: The study was approved by the Danish Data Protection Agency (AHH-2014-042). According to the Danish regulations, anonymized registry studies are not subjected to ethical review.

Consent of publication: Not applicable. 
Data availability: Detailed data can be shared upon request for meta-analyses or other scientific purposes.

Conflict of interest: The authors have no conflicts of interest.

\section{References}

Carethes JM, Stoffel EM. Lynch syndrome and Lynch syndrome mimics: the growin complex landscape of hereditart colon cancer. World J Gastroenterol 2015, 21, 9253-9261.

Choi Y-H, Lakhal-Chaieb L, Kröl A, Yu B, Buchanan D, Ahnen D et al. Risks of colorectal cancer and cancerrelated mortality in familial colorectal cancer type $X$ and Lynch syndrome families. $J$ Nat/ Cancer Inst 2019; e-pub ahead of print 30 October 2018; doi: 10.1093/jnci/djy159.

Engel G, Ferlay J, Cristensen N, Kejs AMT, Johannesen TB, Khan S et al. NORDCAN: Cancer Incidence, Mortality, Prevalence and Survival in the Nordic Countries, Version 7.0 (17.12.2014). Association of the Nordic Cancer Registries, Danish Cancer Society, 2016, http://www.ancr.nu.

Engel C, Loeffler M, Steinke V, Rahner N, Holinski-Feder E, Dietmaier W et al. Risks of less common cancers in proven mutation carriers with lynch syndrome. J Clin Oncol 2012, 30, 4409-4415.

Giardiello FM, Allen JI, Axilbund JE, Boland CR, Burke CA, Burt RW et al. Guidelines on genetic evaluation and management of Lynch syndrome: a consensus statement by the US Multi-society Task Force on colorectal cancer. Am J Gastroentero/ 2014, 109, 1159-1179.

Hampel H, Stephens JA, Pukkala E, Sankila R, Aaltonen LA, Mecklin JP et al. Cancer risk in hereditary nonpolyposis colorectal cancer syndrome: later age of onset. Gastroenterology 2005, 129, 415-421.

Hatfield E, Green JS, Woods MO, Warden G, Parfrey PS. Impact of colonoscopic screening in Familial Colorectal Cancer Type X. Mol Genet Genomic Med. 2018, 6, 1021-1030.

Helder-Woolderink JM, Blok EA, Vasen HF, Hollema H, Mourits MJ, De Bock GH. Ovarian cancer in Lynch syndrome; a systematic review. Eur J Cancer 2016, 55, 65-73.

Jarvinen HJ, Renkonen-Sinisalo L, Aktan-Collan K, Peltomaki P, Aaltonen LA, Mecklin JP. Ten years after mutation testing for Lynch syndrome: cancer incidence and outcome in mutation-positive and mutationnegative family members. J Clin Oncol 2009, 27, 4793-4797.

Joost $\mathrm{P}$, Therkildsen C, Dominguez-Valentin M, Jonsson M, Nilbert M. Urinary Tract Cancer in Lynch Syndrome; Increased Risk in Carriers of MSH2 Mutations. Urology 2015, 86, 1212-1217.

Kastrinos F, Mukherjee B, Tayob N, Wang F, Sparr J, Raymond VM et al. Risk of pancreatic cancer in families with Lynch syndrome. JAMA 2009, 302, 1790-1795. 
Ku CS, Cooper DN, Wu M, Roukos DH, Pawitan Y, Soong R et al. Gene discovery in familial cancer syndromes by exome sequencing: prospects for the elucidation of familial colorectal cancer type X. Mod Pathol. 2012, 25,1055-1068.

Lindberg LJ, Ladelund S, Frederiksen BL, Smith-Hansen L, Bernstein I. Outcome of 24 years national surveillance in different hereditary colorectal cancer subgroups leading to more individualised surveillance. J Med Genet 2016, 54, 297-304.

Lindor NM, Rabe K, Petersen GM, Haile R, Casey G, Baron J et al. Lower cancer incidence in Amseterdam-I criteria familes without mismatch repair deficiency; familial colorectal cancer type X. JAMA 2005, 293, 1979-1985.

Lu KH, Daniels M. Endometrial and ovarian cancer in women with Lynch syndrome: update in screening and prevention. Fam Cancer 2013, 12, 273-277.

Meyer LA, Broaddus RR, Lu KH. Endometrial cancer and Lynch syndrome: clinical and pathologic considerations. Cancer Control 2009, 16, 14-22.

Moller P, Seppala T, Bernstein I, Holinski-Feder E, Sala P, Evans DG et al. Cancer incidence and survival in Lynch syndrome patients receiving colonoscopic and gynaecological surveillance: first report from the prospective Lynch syndrome database. Gut 2017, 66, 464-472.

R Core Team (2019) R: A Language and Environment for Statistical Computing. Vienna, Austria: $\mathrm{R}$ Foundation for Statistical Computing: URL https://www.R-project.org/.

Raymond VM, Mukherjee B, Wang F, Huang SC, Stoffel EM, Kastrinos F et al. Elevated risk of prostate cancer among men with Lynch syndrome. J Clin Oncol 2013, 31, 1713-1718.

Rostgaard K. Methods for stratification of person-time and events - a prerequisite for Poisson regression and SIR estimation. Epidemiol Perspect Innov 2008, 5, 7.

Ryan S, Jenkins MA, Win AK. Risk of prostate cancer in Lynch syndrome: a systematic review and metaanalysis. Cancer Epidemiol Biomarkers Prev 2014, 23, 437-449.

Therkildsen C, Ladelund S, Smith-Hansen L, Lindberg LJ, Nilbert M. Towards a gene- and gender-based risk estimates in Lynch syndrome; age-specific incidences for 13 extra-colorectal cancer types. $\mathrm{Br} \mathrm{J}$ Cancer 2017, 117, 1702-1710.

Valle L. Genetic predisposition to colorectal cancer: where we stand and future perspectives. World J Gastroenterol. 2014, 20, 9828-9849.

Vasen HF, Blanco I, Aktan-Collan K, Gopie JP, Alonso A, Aretz S et al. Revised guidelines for the clinical management of Lynch syndrome (HNPCC): recommendations by a group of European experts. Gut 2013, $62,812-823$. 
Warden G, Harnett D, Green J, Wish T, Woods MO, Green R et al. A population-based study of hereditary non-polyposis colorectal cancer: evidence of pathologic and genetic heterogeneity. Clin Genet. 2013, 84, 522-530.

Win AK, Lindor NM, Young JP, Macrae FA, Young GP, Williamson E et al. Risks of primary extracolonic cancers following colorectal cancer in lynch syndrome. J Natl Cancer Inst 2012, 104, 1363-1372.

Zetner DB, Bisgaard ML. Familial colorecal cancer type X. Curr Genomics 2017, 18, 341-359.

\section{Tables}

Due to technical limitations, the tables are only available as a download in the supplemental files section.

\section{Figures}



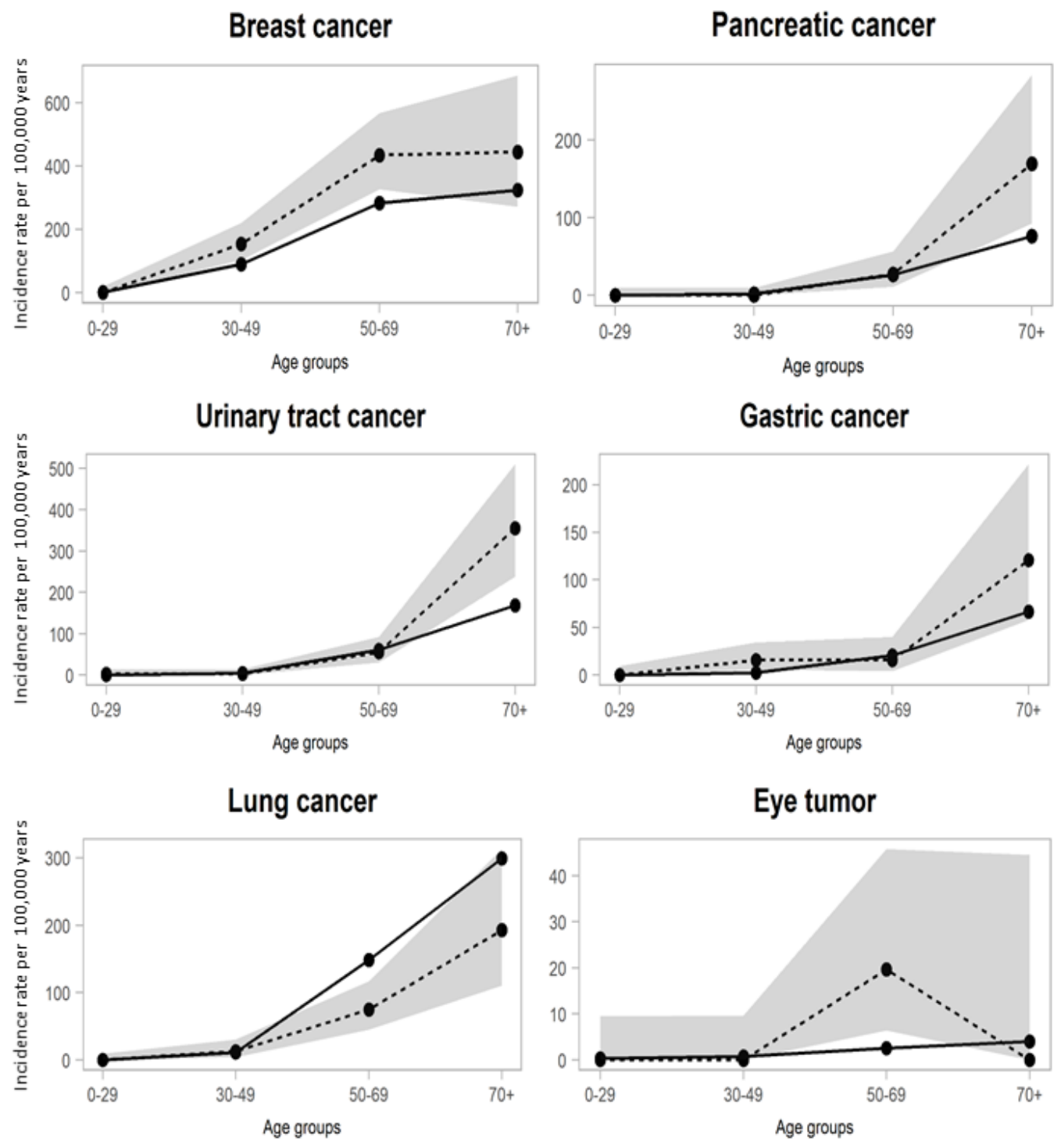

\section{Figure 1}

Age-dependent incidence rates and 95\% confidence intervals for five cancer types with significantly increased incidence rate ratios in at least one age group in FCCTX (dotted lines) compared to the general population (solid lines).

\section{Supplementary Files}


This is a list of supplementary files associated with this preprint. Click to download.

- Supplementarytable1.pdf

- Supplementarytable2.pdf

- Supplementarytable3.pdf

- Table3.xlsx

- Supplementarytable4.pdf

- Supplementaryfigure1.pdf

- Table1.xlsx

- Table2.xlsx 